\title{
FDG-PET and MRI in the Evolution of New-Onset Refractory Status Epilepticus
}

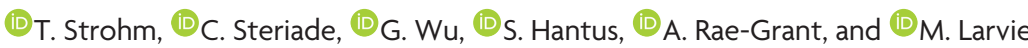

\begin{abstract}
BACKGROUND AND PURPOSE: New-onset refractory status epilepticus is a clinical condition characterized by acute and prolonged pharmacoresistant seizures without a pre-existing relevant neurologic disorder, prior epilepsy, or clear structural, toxic, or metabolic cause. New-onset refractory status epilepticus is often associated with antineuronal antibodies and may respond to early immunosuppressive therapy, reflecting an inflammatory element of the condition. FDG-PET is a useful diagnostic tool in inflammatory and noninflammatory encephalitis. We report here FDG-PET findings in new-onset refractory status epilepticus and their correlation to disease activity, other imaging findings, and outcomes.
\end{abstract}

MATERIALS AND METHODS: Twelve patients who met the criteria for new-onset refractory status epilepticus and who had FDG-PET and MR imaging scans and electroencephalography at a single academic medical center between 2008 and 2017 were retrospectively identified. Images were independently reviewed by 2 radiologists specialized in nuclear imaging. Clinical characteristics and outcome measures were collected through chart review.

RESULTS: Twelve patients underwent 21 FDG-PET scans and 50 MR imaging scans. Nine (75\%) patients were positive for autoantibodies. All patients had identifiable abnormalities on the initial FDG-PET in the form of hypermetabolism (83\%) and/or hypometabolism (42\%). Eight (67\%) had medial temporal involvement. All patients $(n=3)$ with $N$-methyl-D-aspartic acid receptor antibodies had profound bilateral occipital hypometabolism. Initial MR imaging findings were normal in $6(50 \%)$ patients. Most patients had some degree of persistent hyper(73\%) or hypometabolism (45\%) after immunosuppressive therapy. FDG-PET hypometabolism was predictive of poor outcome (mRS 4-6) at hospital discharge $(P=.028)$.

CONCLUSIONS: Both FDG-PET hypometabolism and hypermetabolism are seen in the setting of new-onset refractory status epilepticus and may represent markers of disease activity.

ABBREVIATIONS: $\mathrm{ALE}=$ autoimmune limbic encephalitis; $\mathrm{EEG}=$ electroencephalography; $\mathrm{GABA}-\mathrm{B}=\gamma$-aminobutyric acid $\mathrm{B} ; \mathrm{LGI}=$ leucine-rich glioma inactivated 1; NMDA $=\mathrm{N}$-methyl-D-aspartate; NMDA-R $=\mathrm{N}$-methyl-D-aspartate receptor; $\mathrm{NORSE}=$ new-onset refractory status epilepticus; $\mathrm{VGKC}=$ voltage-gated potassium channel

$\mathbf{N}$ ew-onset refractory status epilepticus (NORSE) is a clinical condition characterized by prolonged pharmacoresistant seizures in an individual without active epilepsy or other pre-

Received July 15, 2018; accepted after revision November 19.

From the Department of Neurology (T.S.), Neurovascular Stroke Center, Ohio State University, Columbus, Ohio; Department of Epilepsy (C.S., S.H.), Department of Nuclear Medicine (G.W., M.L.), Department of Neuroradiology (M.L.), and Department of Neuroimmunology (A.R.-G.), Cleveland Clinic, Cleveland, Ohio. Dr. Strohm was formerly at Cleveland Clinic.

Previously presented as a poster at: Annual Meeting of the Neurocritical Care Society, October 9-13, 2017; Waikoloa, Hawaii.

Please address correspondence to Tamara Strohm, MD, Department of Neurology, Neurovascular Stroke Center, Ohio State University, 3172A Graves Hall, 333 W 10th Ave, Columbus, OH 43210; e-mail: tamara.strohm@gmail.com

三 Indicates article with supplemental on-line tables.

http://dx.doi.org/10.3174/ajnr.A5929 existing relevant neurologic disorders. ${ }^{1,2}$ Other terms such as acute encephalitis with refractory, repetitive partial seizures; devastating epileptic encephalopathy in school-aged children; and febrile infection-related epilepsy syndrome describe the same disease entity. Although the absence of a clear acute or active toxic, metabolic, or structural cause is necessary for the diagnosis, the neuropathology of NORSE includes patients with viral infections (such as herpes simplex virus 1) and autoimmune syndromes such as autoimmune limbic encephalitis (ALE). ${ }^{2-5}$ Typical findings include the following: hippocampal or neocortical T2-hyperintense MR imaging lesions, mild pleocytosis and/or elevated protein in the CSF, and periodic and epileptiform discharges on electroencephalography (EEG). ${ }^{1,6}$ An underlying inflammatory mechanism has been suggested by the following findings: 1) Up to two-thirds of patients are found to have an associated neuronal 
cell surface or intracellular antibody, ${ }^{1} 2$ ) a proportion of patients may respond to early immunosuppressive therapy, ${ }^{7-11}$ and 3) elevated levels of cytokines interleukin- 6 and interleukin- 8 return to normal after treatment, corresponding to clinical improvement. ${ }^{12}$ Given the substantial morbidity (neurologic disability, subsequent epilepsy) and mortality associated with the diagnosis of NORSE, this hypothesis is of interest. The therapeutic implications, in the setting of prolonged intensive care unit and hospital stays and frequent anesthetic treatment, highlight a need for the development of biomarkers to track disease activity and response to therapies.

Abnormal findings on FDG-PET have been demonstrated in conditions such as ALE associated with a NORSE syndrome; however, typical findings and their relevance to disease activity and outcomes have not been established. ${ }^{13,14}$ Meanwhile, the use of FDG-PET is well-established in chronic epilepsy, with interictal hypometabolism suggesting an abnormal function of neuronal networks. ${ }^{15-17}$

We sought to describe FDG-PET characteristics and association of FDG-PET findings with MR imaging, EEG, clinical course, treatment, and outcome measures in a cohort of patients with NORSE.

\section{MATERIALS AND METHODS Patient Selection}

We retrospectively identified patients at a single academic institution from November 2008 to July 2017 in an ALE data base containing patients with autoimmune encephalitis, rapidly progressive dementia, and unexplained new-onset seizures. The data base was searched using the keyword "seizures." Among 26 patients with seizures, 12 patients were identified as fulfilling the consensus definition of NORSE, ${ }^{2}$ all of whom had undergone both FDG-PET and MR imaging. The mean age was 40 years (range, $14-78$ years), and 10 (83\%) patients were female.

Antineuronal antibody panels were performed from serum and/or CSF of all patients (7 [58\%] with both) by commercial laboratories (Athena Laboratories, ARUP; Mayo Medical Laboratories, Rochester, Minnesota) and included a combination of the following: glutamic acid decarboxylase 65; voltage-gated potassium channel (VGKC); leucine-rich glioma inactivated 1 (LGI1); contactin-associated protein $2 ; \gamma$-aminobutyric acid B (GABA-B); $\alpha$-amino-3-hydroxy-5-methyl-4-isoxazolepropionic acid; $N$-methyl-D-aspartate (NMDA); antineuronal nuclear antibody 1, 2, 3; antiglial nuclear antibody 1, 2; Purkinjecell antibody 1, 2; and amphiphysin and anti-Tr antibodies. Eleven (92\%) had NMDA antibody testing, and all patients had VGKC antibody testing. EEG was performed within 24 hours of FDG-PET in 11 (92\%) patients. All patients received immunosuppressive therapy, with 11 (92\%) undergoing FDG-PET following treatment.

The electronic medical record was reviewed for patient characteristics, hospital course, results of diagnostic testing, treatment, and outcome at hospital discharge and last follow-up appointment. Three patients did not have follow-up after hospital discharge.

Approval from the Cleveland Clinic IRB was obtained, and consent was waived due to the retrospective nature of this study.

\section{FDG-PET Imaging}

$\left[{ }^{18} \mathrm{~F}\right]$ FDG (185-370 MBq) was injected intravenously. Imaging was performed approximately 45-60 minutes later using a Biograph 6 PET/CT scanner (Siemens, Erlangen, Germany). The PET emission scan was obtained using a 15-minute acquisition with 1 bed position. PET images were reconstructed using the following parameters: CT attenuation-correction Fourier rebinning, trim sonogram (brain mode), ordered subset expectation maximization, 6 iterations, 8 subsets, 3 -mm Gaussian filter, matrix size = $168 \times 168$, zoom $=2 \times$, and match CT slice location using 2-mm slice thickness. Reconstructed images were reviewed both with and without attenuation correction. Attenuation-correction CT parameters were the following: extended FOV $=700 \mathrm{~mm}, 130$ $\mathrm{kV}$ (peak), $20 \mathrm{mAs}$, 3.0-mm slice thickness, 2.0-mm slice increment, $3 \times 6 \mathrm{~mm}$ collimation, B31s kernel.

\section{MR Imaging Acquisition}

All MR imaging was performed using clinical protocols. Scans were performed on $1.5 \mathrm{~T}$ and $3 \mathrm{~T}$ scanners (Siemens) with protocols designed to provide comparable imaging results from all scanners used for patients in this study; accordingly, no effort was made to use a single scanner. T1, T2, T2 FLAIR, and diffusionweighted images were routinely obtained. SWI or another susceptibility sequence was performed in most scans. Postcontrast T1-weighted images were obtained following intravenous administration of gadoterate meglumine.

\section{Imaging Review}

All FDG-PET and MR imaging scans were independently reviewed by 2 board-certified radiologists specialized in nuclear imaging. Qualitative evaluation was performed by visual inspection using FDG-PET images coregistered with the most recent MR imaging scan. Semiquantitative analysis was performed using a parametric data base comparison with age-matched control subjects as implemented in syngo.via (Siemens). Abnormal findings were characterized as hypermetabolism or hypometabolism and classified according to the brain structure involved, including lobes and, when appropriate, substructures such as the perirolandic region, hippocampus, amygdala, striatum, and cerebellar hemispheres.

\section{Statistical Analysis}

Descriptive statistics were performed for each variable, including means, medians, and SDs for continuous variables and frequencies for categoric variables. Fisher exact tests (2-tailed) were used for comparisons of categoric variables. Significance was set at $P<.05$.

\section{RESULTS}

\section{Patient Characteristics}

Patient characteristics, presenting clinical features, and serum and CSF findings are summarized in On-line Table 1. Most patients $(75 \%)$ were positive for autoantibodies (GABA-B, $n=1$; glutamic acid decarboxylase $65, n=3 ; N$-methyl-D-aspartate receptor (NMDA-R), $n=3$; VGKC, $n=1$; and LGI1, $n=1$ ), and all patients were treated with immunosuppressive therapy at a median of 12.5 days of hospitalization. Most patients had a pro- 


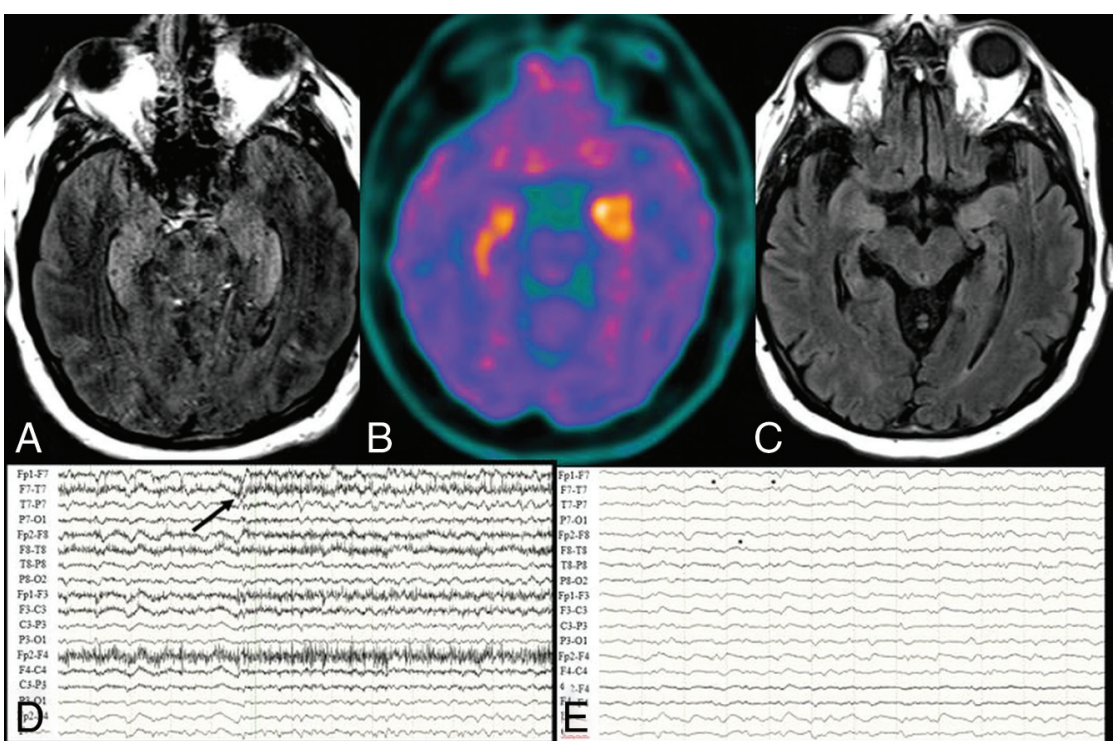

FIG 1. Brain MR imaging, FDG-PET, and EEG findings for P2, a 63-year-old man with glutamic acid decarboxylase 65 encephalitis and normal brain MR imaging findings at the onset of seizures. $A$, Axial T2-weighted FLAIR image at day 12 of status epilepticus shows bilateral hippocampus and insula T2-weighted hyperintense lesions. B, Axial FDG-PET image at day 12 of status epilepticus shows bilateral hippocampus, amygdala, and insula hypermetabolism. C, Axial T2-weighted FLAIR image at day 49 of status epilepticus shows resolution of hippocampal and insula T2-weighted hyperintense lesions with diffuse atrophy. D, EEG shows left frontotemporal seizures (onset at black arrow) with bilateral periodic discharges before seizure. E, EEG shows bilateral independent periodic discharges (black asterisks).

tracted hospital course (median, 48.5 days; range, 19-199 days) with prolonged status epilepticus (median, 12 days, range, 3-106 days). The clinical examination (correlated with the time of MR imaging and FDG-PET) is noted in On-line Table 2. All patients received immunosuppressive therapy (see On-line Table 3 for details).

\section{FDG-PET and MR Imaging Findings}

FDG-PET, MR imaging, EEG, and clinical examination findings are summarized in On-line Table 2 with a highlighted case in Fig 1.

All patients had at least 1 FDG-PET scan, obtained a mean of 13 days after the onset of status epilepticus, (range, $-5-53$ days). All patients had at least 1 abnormal finding on the FDG-PET scan: Seven (58\%) had hypermetabolism only, 2 (17\%) had hypometabolism only, and $3(25 \%)$ had both. Eight (67\%) had bilateral abnormalities, and $6(50 \%)$ had unilateral amygdala and/or hippocampal hypermetabolism. One patient with VGKC-complex antibody and faciobrachial dystonic seizures secondary to a presumed LGIl antibody (not tested) had bilateral basal ganglia hypermetabolism. Three patients (25\%, all with NMDA-R antibodies) had marked bilateral occipital hypometabolism, and 2 of them $(67 \%)$ also had perirolandic hypometabolism. Three patients (27\%) had EEG evidence of seizure at the time of the initial FDG-PET. There was no association between hypermetabolism and EEG seizure at the time of FDG-PET acquisition (Fisher exact test, $P=.49$ ).

Six patients had multiple FDG-PET scans. Of these 6, four (67\%) showed new hypermetabolism and 2 (33\%) had new hypometabolism, so a total of 5 ( $83 \%$ of patients with repeat FDGPET scans) had new abnormalities. Furthermore, of patients with a repeat FDG-PET scan, $4(67 \%)$ had resolution of hypermetabolic foci and 1 (17\%) had resolution of a hypometabolic focus. One complex patient with NMDA-R antibody encephalitis underwent 5 FDG-PET scans from the onset of status epilepticus to day 234 (Fig 2). At seizure onset, his FDG-PET scan showed marked bilateral occipital and parietal hypometabolism, which persisted throughout his disease course.

In comparison, initial MR imaging findings were normal in $6(50 \%)$ patients and remained normal in $3(25 \%)$ patients on a second MR imaging. All patients had abnormal findings by the third MR imaging scan. The most common finding was unilateral amygdala and/or hippocampal T2 hyperintensity, with or without associated swelling. All patients with an initial abnormal finding on MR imaging also had hypermetabolism on the first FDG-PET. No correlation was found between FDG-PET hypometabolism or hypermetabolism and abnormal findings on MR imaging (Fisher exact tests $P=1$ and .45 , respectively).

Other MR imaging findings included superficial cerebral venous thrombosis with or without a small subarachnoid hemorrhage in $2(17 \%)$ and leptomeningeal thickening and enhancement in $2(17 \%)$. Hippocampal or diffuse atrophy or both occurred in $9(75 \%)$ patients, with a median delay from symptom onset to the time of identification of the imaging findings of 48 days (range, 19-937 days).

Eleven patients underwent FDG-PET after immunosuppressive therapy (Fig 3). Eight (73\%) and $5(45 \%)$ patients had hypermetabolism or hypometabolism, respectively, following treatment.

\section{Outcome}

The mean modified Rankin Scale score at hospital discharge was 4 (median, 4; range, 2-5). Nine patients (75\%) followed up at our institution, with a mean follow-up time of 672 days. The mean mRS score improved to 2 (median, 1 ; range, $0-6$ ) at last followup, by which time 5 (56\%) patients had returned to baseline or near-baseline (mRS range, $0-1), 3$ (33\%) had some deficits but remained ambulatory (mRS range, 2-3), and 1 patient had died. Five $(56 \%)$ continued to have clinical seizures at last follow-up and were treated with a mean of 2.5 antiepileptic drugs (range, $1-4)$.

\section{Imaging Predictors of Outcome}

FDG-PET hypometabolism (when seen in the initial FDG-PET obtained) was predictive of poor outcome (defined as mRS 4-6) at hospital discharge (Fisher exact 2-tailed test, $P=.028$ ), while abnormal findings on MR imaging were not predictive of poor outcome at discharge (Fisher exact 2-tailed test, $P=1$ ). No ab- 


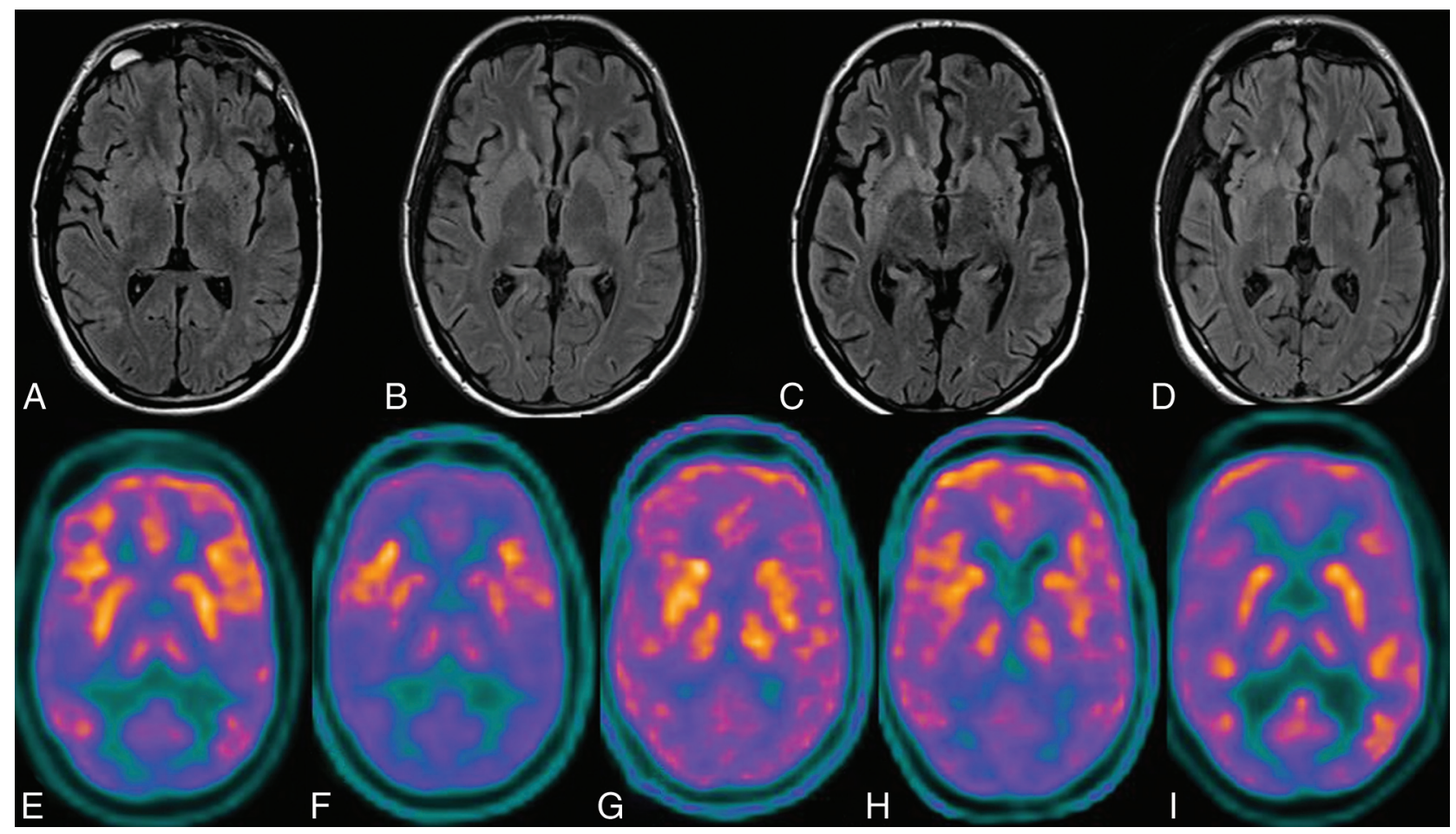

FIG 2. Brain MR imaging and FDG-PET findings for P5, a 22-year-old man with NMDA-R antibody encephalitis. A, Axial T2-weighted FLAIR image 7 days before the onset of status epilepticus with normal findings. $B$, Axial T2-weighted FLAIR image 2 days before the onset of status epilepticus with normal findings. C, Axial T2-weighted FLAIR image at day 28 of status epilepticus shows mild diffuse atrophy. D, Axial T2-weighted FLAIR image at day 63 of status epilepticus shows moderate diffuse atrophy. E, Axial FDG-PET image at day zero of status epilepticus shows marked bilateral occipital-greater than-parietal hypometabolism. F, Axial FDG-PET image at day 12 of status epilepticus shows bilateral inferolateral frontal hypermetabolism with diffuse hypometabolism. G, Axial FDG-PET image at day 56 of status epilepticus shows resolution of bilateral inferolateral frontal hypermetabolism with diffuse cortical hypometabolism. $H$, Axial FDG-PET image at day 88 of status epilepticus shows moderate bilateral occipital-greater than-parietal hypometabolism. I, Axial FDG-PET image at day 234 following status epilepticus shows mild bilateral occipital hypometabolism.

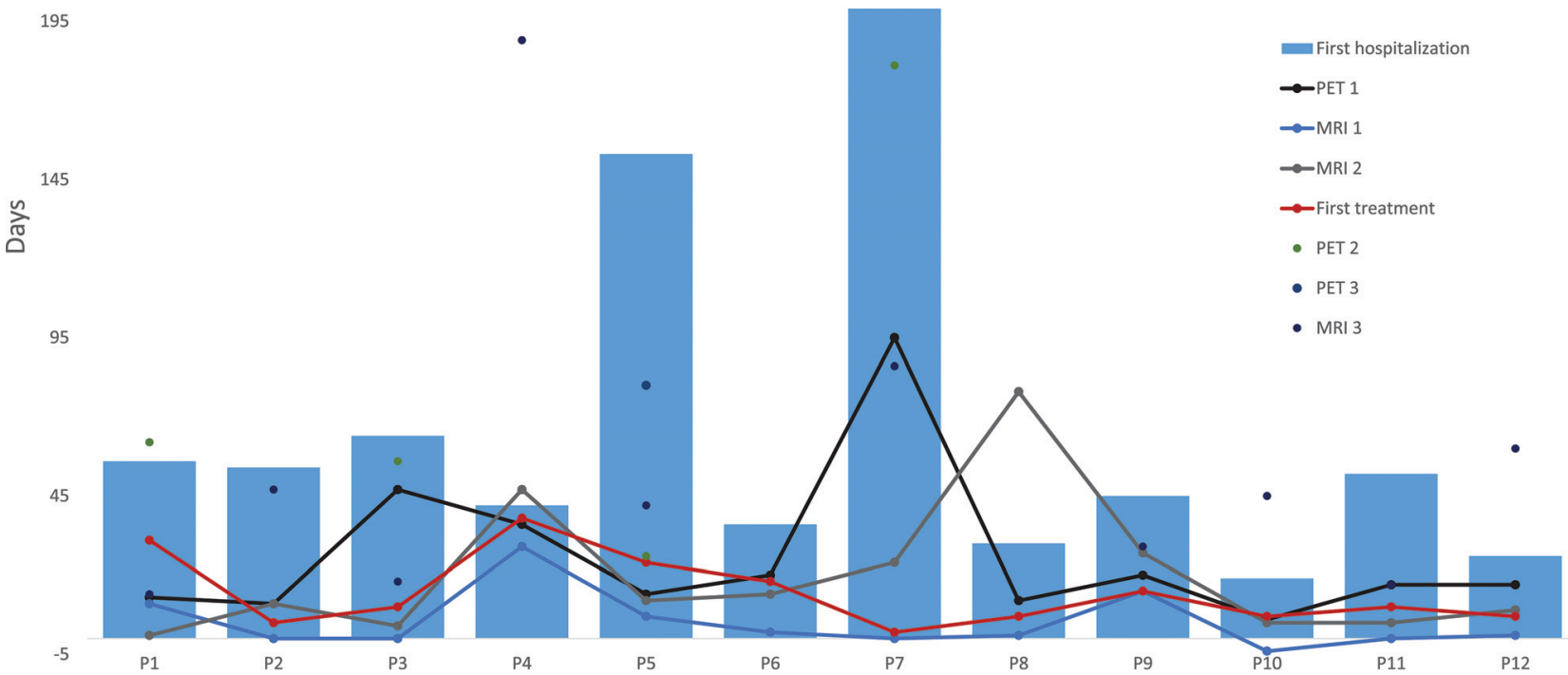

FIG 3. Timeline of brain MR imaging, FDG-PET, and the first immunotherapy treatment during initial hospitalization. Not depicted are FDG-PET 2 of $\mathrm{P} 10$ at 1397 days and MR imaging 3 of P8 at 415 days. 
normal findings on imaging were predictive of persistent seizures at last follow-up. Indeed, we did not find an association between PET hypometabolism (2-tailed Fisher exact test, $P=.52$ ) and abnormal findings on MR imaging (2-tailed Fisher exact test, $P=$ 1) and persistent seizures.

\section{DISCUSSION}

We describe FDG-PET and MR imaging findings spanning the disease course in a cohort of 12 patients with NORSE. We propose a role for FDG-PET as a biomarker of disease activity in NORSE by the following: 1) determining regions of brain vulnerable to encephalitic involvement (amygdala, hippocampus, occipital cortex, sensorimotor cortex, striatum) and discussing the biologic plausibility in the setting of our understanding of mechanisms of NORSE; 2) demonstrating an evolution of FDG-PET findings during the disease course; 3) exploring FDG-PET findings as predictors of outcome; and 4) finally, highlighting a higher proportion of abnormal findings on FDG-PET compared with MR imaging early in the disease course independent of EEG findings.

\section{Regional Vulnerability}

Our findings illustrate a predilection of certain brain regions for abnormal metabolism. The most common regions of metabolic abnormality, as identified on FDG-PET, are the amygdala and hippocampus (67\% of cases), which are also regions where MR imaging abnormalities were frequently identified ( $42 \%$ of cases). In the setting of ALE, medial temporal and limbic hypermetabolism or hypometabolism has also been reported, ${ }^{18,19}$ mirroring abnormalities of the hippocampus and amygdala on MR imaging. ${ }^{20-23}$ This regional predilection on neuroimaging may reflect an underlying neuroinflammatory pathology preferentially affecting the hippocampus, which may be common not only to ALE but also to NORSE. Infiltration of inflammatory cells and complement activation have been shown to lead to parenchymal edema in histopathologic studies of VGKC-antibody-associated ALE, demonstrating limbic T-cell infiltration with perivascular and parenchymal B-cells in the hippocampus. ${ }^{24}$ Regional predilection may reflect antigen expression topography and/or, on a broader level, regional susceptibility to neuroinflammation. ${ }^{25}$ The latter is supported by the finding in this study that FDG-PET medial temporal hypermetabolism was present with multiple types of antibodies (NMDA-R, glutamic acid decarboxylase 65, VGKC, GABA-B) as well as cases negative for antibodies.

Other regional vulnerabilities were found in association with specific antibodies. Bilateral occipital and perirolandic hypometabolism were noted in patients with NMDA-R antibodies, consistent with previous reports of a frontotemporalto-occipital gradient with hypermetabolism in the frontal and temporal lobes and profound hypometabolism in the occipital lobes. ${ }^{13,26,27}$ Similarly, striatal hypermetabolism was noted in 1 patient with VGKC-LGI1, similar to previous reports of abnormalities in the cortical sensorimotor and basal ganglia metabolism in the VGKC-antibody ALE. ${ }^{28}$ Quartuccio et $\mathrm{al}^{29}$ hypothesized that antibodies against intracellular antigens were more likely to cause a T-cell-mediated release of cytokines and chemokines in the limbic system manifesting as FDG-PET hypermetabolism. They proposed that FDG-PET hypometabolism, conversely, was secondary to cell-surface antibody-mediated capping and internalization leading to a decrease in neuronal activity.

Finally, widespread findings without region predilection have been reported in a cohort of children with febrile infection-related epilepsy syndrome, in whom widespread hypometabolism in the bilateral temporoparietal and orbitofrontal cortices was found to correspond with EEG findings and neuropsychological deficits. $^{30}$

\section{Evolution of FDG-PET Findings during the Disease Course}

Most patients with serial FDG-PET and MR imaging scans showed evolution, with resolution of prior findings and new abnormalities on subsequent imaging (Figs 1 and 2). While the main driver of the evolution in FDG-PET findings is unclear, one may hypothesize a role for severity of illness, treatment effect, and underlying pathology. Other findings including leptomeningeal thickening and enhancement, subarachnoid hemorrhage, and cerebral venous thrombosis on MR imaging have been described in ALE. Cerebral venous thrombosis and subarachnoid hemorrhage may reflect abnormalities in the coagulation cascade secondary to an underlying inflammatory process or immunosuppressive therapy.

\section{FDG-PET as a Predictor of Outcome}

FDG-PET abnormalities were correlated with poor outcome at hospital discharge. Conversely, both cases with normal FDG-PET on serial imaging had good (mRS, $0-3$ ) outcomes at hospital discharge. Others have reported normalization of cerebral glucose metabolism with recovery. ${ }^{31}$ The variability in outcomes reported (for instance, 20\% mortality, 30\% debilitated with mRS 4-5, and $50 \%$ with mRS $0-3$ at 1 year in Kantanen et al, ${ }^{32}$ in 2017) may reflect variability in underlying pathophysiology and treatment practices (aggressiveness of anesthetic and barbiturate use, use and escalation of immunosuppressive therapy). At the heart of variation in treatment practices is a lack of biomarkers beyond EEG to guide clinical decision-making. In this cohort, abnormal findings on FDG-PET were present independent of the persistence of EEG seizures. This finding suggests that abnormal FDGPET metabolism may be more directly related to the pathophysiology of the disease process than seizure activity or other metrics routinely followed in clinical practice.

\section{Comparative Value of FDG-PET and MR Imaging as Imaging Modalities in NORSE}

Others have reported increased sensitivity of FDG-PET versus MR imaging during the initial evaluation. ${ }^{29,33,34}$ Our findings mirrored this: Fifty percent of MR imaging scans initially had abnormal findings compared with $100 \%$ of FDG-PET scans. With time, an increasing proportion of MRIs showed some abnormal findings. The higher chance of an abnormality on an FDGPET scan early in the disease course not only increases its diagnostic value but also raises the possibility that it may be a more useful biomarker. 


\section{Limitations}

Limitations include the retrospective nature of this study, small sample size, and heterogeneity in patient characteristics. Given that NORSE is a clinical diagnosis, diversity among autoantibodies and disease processes may limit the ability to generalize. Excepting cases with NMDA-R antibodies, we found that abnormal findings on FDG-PET were not segregated by antibody type or seropositive groups. Additionally, the retrospective nature of the study was associated with variability in the timing of FDG-PET and MR imaging scans. The variability in treatment received, both antiepileptic including barbiturate and immunotherapeutic, could also have impacted glucose metabolism on FDG-PET.

\section{CONCLUSIONS}

Evolving patterns of brain hypermetabolism and hypometabolism, as revealed by FDG-PET, are seen in NORSE, with a predilection for the amygdala and hippocampus in cases with a diversity of autoantibodies. In the cohort presented here, FDG-PET often showed patterns of mixed hypermetabolism and hypometabolism in the earlier, more acute phases, with evolution to hypometabolism in the later, more chronic phases. We propose an independent role for FDG-PET as a complementary marker of disease activity in NORSE.

\section{REFERENCES}

1. Gaspard N, Foreman BP, Alvarez V, et al; Critical Care EEG Monitoring Research Consortium (CCEMRC). New-onset refractory status epilepticus: etiology, clinical features, and outcome. Neurology 2015;85:1604-13 CrossRef Medline

2. Hirsch LJ, Gaspard N, van Baalen A, et al. Proposed consensus definitions for new-onset refractory status epilepticus (NORSE), febrile infection-related epilepsy syndrome (FIRES), and related conditions. Epilepsia 2018;59:739-44 CrossRef Medline

3. Graus F, Titulaer MJ, Balu R, et al. A clinical approach to diagnosis of autoimmune encephalitis. Lancet Neurol 2016;15:391-404 CrossRef Medline

4. Ismail FY, Kossoff EH. AERRPS, DESC, NORSE, FIRES: Multi-labeling or distinct epileptic entities? Epilepsia 2011;52:e185-89 CrossRef Medline

5. Sakuma H, Awaya Y, Shiomi M, et al. Acute encephalitis with refractory, repetitive partial seizures (AERRPS): a peculiar form of childhood encephalitis. Acta Neurol Scand 2010;121:251-56 CrossRef Medline

6. Costello DJ, Kilbride RD, Cole AJ. Cryptogenic new onset refractory status epilepticus (NORSE) in adults: infectious or not? J Neurol Sci 2009;277:26-31 CrossRef Medline

7. Byun J, Lee S, Jung K, et al. Effect of immunotherapy on seizure outcome in patients with autoimmune encephalitis: a prospective observational registry study. PLoS One 2016;11:e0146455 CrossRef Medline

8. Gall CR, Jumma O, Mohanraj R. Five cases of new onset refractory status epilepticus (NORSE) syndrome: outcomes with early immunotherapy. Seizure 2013;22:217-20 CrossRef Medline

9. Iizuka T, Kanazawa N, Kaneko J, et al. Cryptogenic NORSE: its distinctive clinical features and response to immunotherapy. Neurol Neuroimmunol Neuroinflamm 2017;4:e396 CrossRef Medline

10. Khawaja AM, DeWolfe JL, Miller DW, et al. New-onset refractory status epilepticus (NORSE): the potential role for immunotherapy. Epilepsy Behav 2015;47:17-23 CrossRef Medline

11. Li J, Saldivar C, Maganti RK. Plasma exchange in cryptogenic new onset refractory status epilepticus. Seizure 2013;22:70-73 CrossRef Medline
12. Kenney-Jung DL, Vezzani A, Kahoud RJ, et al. Febrile infectionrelated epilepsy syndrome treated with anakinra. Ann Neurol 2016; 80:939-45 CrossRef Medline

13. Leypoldt F, Buchert R, Kleiter I, et al. Fluorodeoxyglucose positron emission tomography in anti-N-methyl-D-aspartate receptor encephalitis: distinct pattern of disease. J Neurol Neurosurg Psychiatry 2012;83:681-86 CrossRef Medline

14. Titulaer M, Soffietti R, Dalmau J, et al; European Federation of Neurological Societies. Screening for tumours in paraneoplastic syndromes: report of an EFNS task force. Eur J Neurol 2011;18:19-e3 CrossRef Medline

15. Cole AJ. Status epilepticus and periictal imaging. Epilepsia 2004;45: 72-77 Medline

16. Kim S, Mountz JM. SPECT imaging of epilepsy: an overview and comparison with F-18 FDG PET. Int J Mol Imaging 2011;2011: 813028 CrossRef Medline

17. Sarria-Estrada S, Toledo M, Lorenzo-Bosquet C, et al. Neuroimaging in status epilepticus secondary to paraneoplastic autoimmune encephalitis. Clin Radiol 2014;69:795-803 CrossRef Medline

18. Baumgartner A, Rauer S, Mader I, et al. Cerebral FDG PET and MRI findings in autoimmune limbic encephalitis: correlation with autoantibody types. J Neurol 2013;260:2744-53 CrossRef Medline

19. Probasco JC, Solnes L, Nalluri A, et al. Abnormal brain metabolism on FDG PET/CT is a common early finding in autoimmune encephalitis. Neurol Neuroimmunol Neuroinflamm 2017;4:e352 CrossRef Medline

20. Finke C, Prüss H, Heine J, et al. Evaluation of cognitive deficits and structural hippocampal damage in encephalitis with leucine-rich, glioma-inactivated 1 antibodies. JAMA Neurol 2017;74:50-59 CrossRef Medline

21. Heine J, Prüss $\mathrm{H}$, Bartsch $\mathrm{T}$, et al. Imaging of autoimmune encephalitis-relevance for clinical practice and hippocampal function. Neuroscience 2015;309:68-83 CrossRef Medline

22. Miller TD, Chong TT, Aimola Davies AM, et al. Focal CA3 hippocampal subfield atrophy following LGI1 VGKC-complex antibody limbic encephalitis. Brain 2017;140:1212-19 CrossRef Medline

23. Wagner J, Schoene-Bake J, Malter MP, et al. Quantitative FLAIR analysis indicates predominant affection of the amygdala in antibody-associated limbic encephalitis. Epilepsia 2013;54:1679-87 CrossRef Medline

24. Khan NL, Jeffree MA, Good C, et al. Histopathology of VGKC antibody-associated limbic encephalitis. Neurology 2009;72:1703-05 CrossRef Medline

25. Vincent A, Bien CG, Irani SR, et al. Autoantibodies associated with diseases of the CNS: new developments and future challenges. Lancet Neurol 2011;10:759-72 CrossRef Medline

26. Maeder-Ingvar M, Prior JO, Irani SR, et al. FDG PET hyperactivity in basal ganglia correlating with clinical course in anti-NDMA-R antibodies encephalitis. J Neurol Neurosurg Psychiatry 2011;82:235-36 CrossRef Medline

27. Probasco JC, Solnes L, Nalluri A, et al. Decreased occipital lobe metabolism by FDG PET/CT: an antip-NMDA receptor encephalitis biomarker. Neurol Neuroimmunol Neuroinflamm 2018;5:e413 CrossRef Medline

28. Dodich A, Cerami C, Iannaccone S, et al. Neuropsychological and FDG-PET profiles in VGKC autoimmune limbic encephalitis. Brain Cogn 2016;108:81-87 CrossRef Medline

29. Quartuccio N, Caobelli F, Evangelista L, et al; Young AIMN Working Group. The role of FDG PET/CT in the evaluation of patients affected by limbic encephalitis: a systematic review of the literature. J Neuroimmunol 2015;284:44-48 CrossRef Medline

30. Mazzuca M, Jambaque I, Hertz-Pannier L, et al. 18F-FDG PET reveals frontotemporal dysfunction in children with fever-induced refractory epileptic encephalopathy. J Nucl Med 2011;52:40-47 CrossRef Medline

31. Nardetto L, Zoccarato M, Santelli L, et al. (18)F-FDG PET/MRI in cryptogenic new-onset refractory status epilepticus: a potential 
marker of disease location, activity and prognosis? J Neurol Sci 2017; 381:100-02 CrossRef Medline

32. Kantanen A, Reinikainen M, Parviainen I, et al. Long-term outcome of refractory status epilepticus in adults: a retrospective population-based study. Epilepsy Res 2017;133:13-21 CrossRef Medline

33. Solnes LB, Jones KM, Rowe SP, et al. Diagnostic value of 18F-FDG
PET/CT versus MRI in the setting of antibody-specific autoimmune encephalitis. J Nucl Med 2017;58:1307-13 CrossRef Medline

34. Newey C, Sarwal A, Hantus S. [18F]-fluoro-deoxy-glucose positron emission tomography scan should be obtained early in cases of autoimmune encephalitis. Autoimmune Dis 2016;2016:9450452 CrossRef Medline 